\title{
CCD IMAGING OF PLANETARY NEBULA HALOS
}

\author{
K.B. KWITTER \\ Astronomy Dept., Williams College, Williamstown, MA 01267 USA \\ Y.-H. CHU \\ Astronomy Dept., University of Illinois, Urbana, IL 61821 USA \\ and \\ R.A. DOWNES \\ Space Telescope Science Institute, Baltimore, MD 21218 USA
}

We have obtained deep CCD images of $14 \mathrm{PN}$ to search for and examine faint halos. These images were obtained with $\mathrm{H} \beta$, [OIII], $\mathrm{H} \alpha$, and [NII] narrowband interference filters on the Burrell Schmidt at Kitt Peak. Table 1 summarizes the observations. We have found interesting features around NGC 1360, NGC 3587 (The Owl), and NGC 6853 (The Dumbbell), and we have obtained new images of the very faint outer halo of NGC 7293 (The Helix). Two CCDs were used: TI6, an $800 \times 800$ chip, with $1.45 \% /$ pix and a 19.3 arcmin square field., and ST2K, a $2048 \times 2048$ chip, of which only $1200 \times 1200$ pixels were read out, yielding $2.07 \%$ pix and a 40 arcmin square field.

TABLE 1. Planetary Nebulae Observed

\begin{tabular}{|c|c|c|c|c|c|}
\hline Nebula & Chip & Filters & Nebula & Chip & Filters \\
\hline$N G C, 40$ & $\overline{\text { TI6 }}$ & $\mathrm{H} \beta,[\mathrm{OIII}], \mathrm{H} \alpha,[\mathrm{NII}]$ & NGC 6309 & $\overline{\mathrm{ST} 2 \mathrm{~K}}$ & [OIII], $\mathrm{H} \alpha,[\mathrm{NII}]$ \\
\hline NGC: 246 & ST $2 \mathrm{~K}$ & $\mathrm{H} \alpha$ & NGC 6543 & ST2K & {$[\mathrm{OIII}], \mathrm{H} \alpha,[\mathrm{NII}]$} \\
\hline NGC $650-1$ & ST2K & $\mathrm{H} \alpha,[\mathrm{OIII}]$ & NGC 6853 & TI6 & $\mathrm{H} \beta,[\mathrm{OIII}], \mathrm{H} \alpha,[\mathrm{NII}]$ \\
\hline$N G C: 1360$ & ST $2 \mathrm{~K}$ & [OIII], $\mathrm{H} \alpha,[\mathrm{NII}]$ & NGC 6720 & ST2K & [OIII] $, \mathrm{H} \alpha,[\mathrm{NII}]$ \\
\hline NGC: 1514 & TI6 & $\mathrm{H} \beta,[\mathrm{OIII}], \mathrm{H} \alpha,[\mathrm{NII}]$ & NGC 7293 & ST2K & {$[\mathrm{OIII}], \mathrm{H} \alpha,[\mathrm{NII}]$} \\
\hline NGC 2022 & TI6 & $\mathrm{H} \beta,[\mathrm{OIII}], \mathrm{Ha},[\mathrm{NII}]$ & NGC 7354 & TI6 & [OIII] \\
\hline NGC: 3587 & ST2K & [OIII] $, \mathrm{H} \alpha,[\mathrm{NII}]$ & NGC 7662 & TI6/ST2K & {$[\mathrm{OIII}] / \mathrm{H} \beta, \mathrm{H} \alpha,[\mathrm{NII}]$} \\
\hline
\end{tabular}

Figure 1 is NGC 1360 in [OIII]; the lobes projecting to the northeast and southwest are also visible in $\mathrm{H} \alpha$ and [NII]. Figure 2 is NGC 3587 in [OIII]; the off-center, round halo seen here is also visible, though not nearly as prominent, in $\mathrm{H} \alpha$ and [NII]. Figure 3 is NGC 6853 in [OIII]; the extended, almost bipolar halo is also evident, though clumpier, in $\mathrm{H} \alpha$ and [NII] (Kwitter et al. 1991). Figure 4 is NGC 7293 in $\mathrm{H} \alpha$; the scalloped projections to the east and the diffuse clump to the northeast were first noticed by Malin (1982). The clump is also bright in [NII]. More detailed analysis of these halos is in progress (Kwitter et al. 1992).

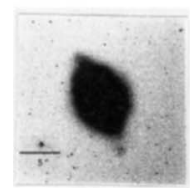

Fig. 1 NGC1360-[OIII]

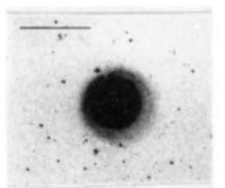

Fig. 2 NGiC3587-[OII]

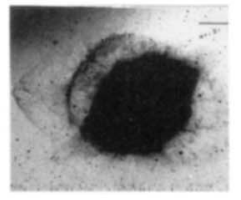

Fig. 3 NGC $7293-\mathrm{H} \alpha$

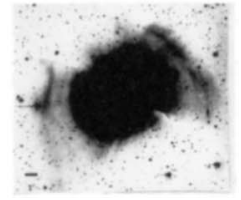

Fig. 4 NGC6853-[OIII]

\section{References}

Kwitter, K.B., Downes, R.A., and Chu., Y.-H. 1991, "Schmidt CCD Images of the Dumbbell Nebula (NGC 6853),"

Bulletin of the A.A.S., 23, 914.Kwitter, K.B., Chu., Y.-H., and Downes, R.A. 1992, "A Study of Planetary Nebula Halos," in prepara tion.

Malin, D.F. 1982, "A Look at Some Unstable Stars," Sky and Telescope, 63, 22. 